\title{
The application of hyaluronic acid-derivatized carbon nanotubes in hematoporphyrin monomethyl ether-based photodynamic therapy for in vivo and in vitro cancer treatment
}

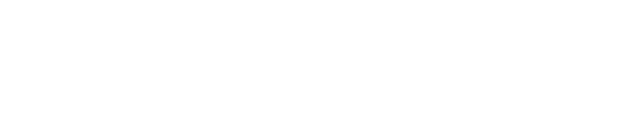

Jinjin Shi*

Rourou Ma*

Lei Wang

Jing Zhang

Ruiyuan Liu

Lulu Li

Yan Liu

Lin Hou

Xiaoyuan Yu

Jun Gao

\section{Zhenzhong Zhang}

School of Pharmaceutical Sciences, Zhengzhou University, Zhengzhou, Henan, People's Republic of China

*These authors contributed equally to this work

Correspondence: Zhenzhong Zhang School of Pharmaceutical Sciences, Zhengzhou University, 100 Kexue Avenue, Zhengzhou, Henan 45000 I, People's Republic of China

$\mathrm{Tel}+8637 \mid 6778$ I9I0

Fax +86 37I 67781908

Email zhangzz08@।26.com

\begin{abstract}
Carbon nanotubes (CNTs) have shown great potential in both photothermal therapy and drug delivery. In this study, a CNT derivative, hyaluronic acid-derivatized CNTs (HA-CNTs) with high aqueous solubility, neutral $\mathrm{pH}$, and tumor-targeting activity, were synthesized and characterized, and then a new photodynamic therapy agent, hematoporphyrin monomethyl ether (HMME), was adsorbed onto the functionalized CNTs to develop HMME-HA-CNTs. Tumor growth inhibition was investigated both in vivo and in vitro by a combination of photothermal therapy and photodynamic therapy using HMME-HA-CNTs. The ability of HMME-HA-CNT nanoparticles to combine local specific photodynamic therapy with external near-infrared photothermal therapy significantly improved the therapeutic efficacy of cancer treatment. Compared with photodynamic therapy or photothermal therapy alone, the combined treatment demonstrated a synergistic effect, resulting in higher therapeutic efficacy without obvious toxic effects to normal organs. Overall, it was demonstrated that HMME-HA-CNTs could be successfully applied to photodynamic therapy and photothermal therapy simultaneously in future tumor therapy.

Keywords: photodynamic therapy, photothermal therapy, HA-derivatized carbon nanotubes, tumor targeting, synergistic effect, hematoporphyrin monomethyl ether
\end{abstract}

\section{Introduction}

Carbon nanotubes (CNTs) are promising materials due to their unique structures and outstanding physical and chemical properties, ${ }^{1}$ thus offering an extensive application in many disciplines, such as engineering, biology, chemistry, physics, and medicine. Concerning drug delivery, therapeutic components for drugs, ${ }^{2,3}$ peptides, ${ }^{4}$ proteins, genes, ${ }^{5}$ and small interfering RNA ( porous absorbance, functional attachment and encapsulation ${ }^{5}$. However, the insolubility in most solvents and high cytotoxic of pristine $\mathrm{CNTs}^{7}$ limited their potential utility in biomedicine. It has been an active and significant direction of research to functionalize CNTs to improve their water-solubility, which can be achieved by functionalization with carboxyl group, ${ }^{5}$ phospholipids, ${ }^{8}$ and polyethylene glycol. ${ }^{2,9}$ CNTs have shown great potential in cancer treatments because of their controlled and targeted drug delivery, which can simultaneously lead to higher therapeutic efficiency and minimize side effects. ${ }^{10}$ Besides, CNTs have high optical absorbance in the near-infrared (NIR) region, where biological systems have low absorption and high transparency, ${ }^{11}$ resulting in heating of CNTs, ${ }^{12}$ thus offering a possibility for cancer photothermal therapy. ${ }^{13}$ 
Hyaluronic acid (HA) is a naturally occurring linear polysaccharide with negative charge, which exists widely in extracellular matrix and is primarily cleared by the lymphatic system. ${ }^{14}$ HA has a series of excellent properties, such as biocompatible, biodegradable, and nonimmunogenic characteristics. ${ }^{15,16}$ The expression of receptors for HA was reported to have a close relation with the occasion of tumors. ${ }^{17}$ Many tumor cells show upregulated expression of receptors for HA, resulting in HA having a high affinity for tumors. ${ }^{18}$ Normal cells also express CD44, but the receptors of normal cells remain in a condition of inactivity and silence, ${ }^{19}$ thus offering HA good selectivity of tumor cells. HA-based tumor-targeted drug delivery has been an emerging and fast-growing field. It has been demonstrated that HA nanoparticles showed rapid uptake into tumor cells and can enter tumor cells through receptor-mediated endocytosis, based on the interactions between the HA of nanoparticles and CD44 of tumor cells. ${ }^{20}$ In this study, HA was linked to CNTs in order to obtain a water-soluble and tumor-targeting drug carrier - HA-CNTs.

Photodynamic therapy (PDT) has been under investigation as a treatment for cancer and some other nonmalignant diseases. ${ }^{21}$ It is a promising modality for the management of various tumors and nonmalignant diseases, based on the application of a photosensitizer that is selectively localized in the target tissue, activated by a specific wavelength of light and resulting in photodamage and subsequent cell death. Hematoporphyrin monomethyl ether (HMME) is a new-generation porphyrin-related photosensitizer, with lower toxicity and shorter-term skin photosensitization than the old generations of PDT drugs. ${ }^{22,23}$ However, the poor hydrophilicity of HMME, lack of tumor targeting, and the absorption of light leading to agglomeration in water has limited its clinical use. It has been reported that micro/nanoparticle sizes within a 400-600 nm interval of polylactic acid loaded with HMME can improve the hydrophilicity of HMME, which is prepared by oil-in-water $(\mathrm{O} / \mathrm{W})$ solvent evaporation, and achieved good antitumor effects. ${ }^{24}$ In this work, HMME was used as a model drug, and was loaded onto HA-CNTs to obtain a HMME-HA-CNT drug-delivery system for the purpose of overcoming the aforementioned limits of HMME.

In this study, a CNT derivative, HA-HA-CNTs, with high aqueous solubility, neutral $\mathrm{pH}$, and tumor-targeting activity, was synthesized, characterized, and explored for applications in drug delivery and photothermal therapy. It was found that the PDT agent HMME was adsorbed on the functionalized CNTs by $\pi-\pi$ stacking interaction via noncovalent bond action and achieved high loading efficiency on HA-CNTs. Herein, a tumor-targeting delivery system (HMME-HA-CNTs) was developed and characterized by transmission electron microscopy (TEM), scanning electron microscopy (SEM), and dynamic laser scattering. Moreover, photothermal therapy of CNTs in the NIR region (808 nm) and PDT of HMME in the visible light region $(532 \mathrm{~nm})$ were simultaneously carried out to strengthen the antitumor effects. The synergistic therapeutic efficiency of PDT and photothermal therapy was examined using HA receptor-positive cells (B16F10 mice melanoma cells) in vitro and tumor-bearing mice models (mice melanoma) in vivo.

\section{Materials and methods Materials}

Single-walled CNTs (purity $>90 \%$ ) were purchased from Shenzhen Nanotech Port (Shenzhen, People's Republic of China). HMME (purity $>98 \%$ ) was obtained from Beijing Yi-He Biotech (Beijing, People's Republic of China). HA (purity $>98 \%$, molecular weight 14,000-20,000), ethylenediamine $\left(\mathrm{H}_{2} \mathrm{NCH}_{2} \mathrm{CH}_{2} \mathrm{NH}_{2}\right)$, formamide, dicyclohexylcarbodiimide, $N$-(3-dimethylamino propyl- $N^{\prime}$-ethylcarbodiimide) hydrochloride (EDC $\cdot \mathrm{HCl}$ ), sodium dodecyl sulfate (SDS), fluorescein isothiocyanate (FITC), Hoechst 33258, and dimethyl sulfoxide were obtained from Sigma-Aldrich (St Louis, MO, USA). Sulforhodamine B (SRB), Dulbecco's Modified Eagle's Medium (DMEM), penicillin, streptomycin, fetal bovine serum, and heparin sodium were bought from Life Technologies (Carlsbad, CA, USA). Quantum dots (CdSe/ZnS Qds) were supplied by Wuhan Jiayuan Quantum Dots (Wuhan, People's Republic of China). Other reagents were acquired from China National Medicine Corporation (Beijing, People's Republic of China). The dialysis bags (molecular weight cutoff $=10,000$ ) were from Spectrum Laboratories (Rancho Dominguez, CA, USA).

\section{Synthesis of HA-CNTs}

The CNTs were oxidized by use of nitric acid and using mixture acid, as reported in the literature. ${ }^{25}$ Then, $\mathrm{COOH}-$ CNTs (100 mg) and dicyclohexylcarbodiimide (500 mg) were sonicated in ethylenediamine $\left(\mathrm{H}_{2} \mathrm{NCH}_{2} \mathrm{CH}_{2} \mathrm{NH}_{2}\right.$, $100 \mathrm{~mL}$ ), stirred, and refluxed at $120^{\circ} \mathrm{C}$ under protection of argon gas for 48 hours. The amine-functionalized CNTs $\left(\mathrm{NH}_{2}-\mathrm{CNTs}\right)$ were obtained by filtering and washing in ethanol ten times and deionized water several times. The resulting solid products were dried in vacuum at $60^{\circ} \mathrm{C}$ for 24 hours. Finally, these products $(100 \mathrm{mg})$, HA $(210 \mathrm{mg})$, and $\mathrm{EDC} \cdot \mathrm{HCl}(75 \mathrm{mg})$ were added to formamide $(100 \mathrm{~mL})$, then stirred at room temperature in the dark for 48 hours, 
and finally dialyzed by a membrane (molecular weight cutoff $=10,000$, Spectrum Laboratories) for 3 days to remove free HA and EDC. The resulting product (HA-CNTs) was dried in vacuum at $60^{\circ} \mathrm{C}$ for 24 hours.

\section{HMME adsorption on HA-CNTs}

HA-CNTs (50 mg) were added to an ethanol-water mixture (ethanol: water $=1: 1,50 \mathrm{~mL})$ containing HMME $(150 \mathrm{mg}$ ) and sonicated at room temperature for 2 hours. After evaporation to remove ethanol and water, the product was dispersed in water $(20 \mathrm{~mL})$ and sonicated using an ultrasonic cell-disruption system (400 W, ten times), and then the nanosuspension was centrifuged to remove free HMME. The entire process was carried out in the dark, and the resulting HMME-HA-CNT nanosuspension was stored at $4^{\circ} \mathrm{C}$ in the dark until use.

\section{Characterization}

Dynamic laser scattering (Zetasizer Nano ZS-90; Malvern Instruments, Malvern, UK), TEM (Tecnai G ${ }^{2}$ 20; FEI, Hillsboro, OR, USA) and SEM (JSM6330F; Zeiss, Jena, Germany) were used to characterize particle size, zeta potential, and morphological aspects of the HMME-HA-CNTs, respectively. The optical properties of HA-CNTs and HMMEHA-CNTs were characterized using an ultraviolet (UV)-visible spectrometer (Lambda35; PerkinElmer, Waltham, MA, USA). Fourier-transform infrared (FT-IR) spectra were recorded on a Nicolet iS10 spectrometer (Thermo Fisher Scientific, Waltham, MA, USA). The relative amount of HA linked to CNTs was tested using a thermal gravimetric analysis (TGA; PerkinElmer) with experimental conditions of scanning from $25^{\circ} \mathrm{C}$ to $800^{\circ} \mathrm{C}$ under nitrogen at a heating rate of $20^{\circ} \mathrm{C} /$ minute.

\section{Determination of HMME loading and release}

The HMME-HA-CNT nanosuspension was diluted with anhydrous ethanol and sonicated to ensure that HMME was dissolved completely, then centrifuged to separate HA-CNTs and HMME to determine the amount of HMME loaded onto HA-CNTs. The concentrations of HMME were determined by high-performance liquid chromatography (1100; Agilent Technologies, Santa Clara, CA, USA) with the following conditions: an Eclipse XDB-C18 column (Agilent, $150 \times 4.6 \mathrm{~mm}, 5.0 \mu \mathrm{m})$; mobile-phase sodium acetate solution ( $0.02 \mathrm{~mol} / \mathrm{L})$ :tetrahydrofuran 60:40; column temperature $40^{\circ} \mathrm{C}$; fluorescence detector with the excitation and emission wavelengths set at 395 and $613 \mathrm{~nm}$, respectively; flow rate $1.0 \mathrm{~mL} /$ minute; and injection volume $20 \mu \mathrm{L} .{ }^{26}$
The quantity of CNTs was determined at $808 \mathrm{~nm}$ by UV-visible spectrometer. ${ }^{25}$

For the release study, HMME-HA-CNTs and HMME samples, which had the same HMME concentration, were placed into dialysis bags, which were dialyzed in $50 \mathrm{~mL}$ SDS, $0.5 \%$. The release assay was performed at $37.0^{\circ} \mathrm{C} \pm 0.5^{\circ} \mathrm{C}$ with a stirring speed of 100 revolutions per minute; $0.2 \mathrm{~mL}$ solution was drawn from the dialysis bags at various time points, being replaced by the same volume of fresh SDS. The concentration of HMME released from HA-CNTs into SDS solution was quantified using high-performance liquid chromatography under the aforementioned chromatographic conditions.

\section{Laser irradiation and temperature- measurement study}

For PDT, the visible light source used was a $532 \mathrm{~nm}$ laser emitter, and its light intensity at the treatment site was $100 \mathrm{~mW} / \mathrm{cm}^{2}$. For photothermal therapy, the NIR light source used was a $808 \mathrm{~nm}$ laser emitter, and its light intensity at the treatment site was $1.4 \mathrm{~W} / \mathrm{cm}^{2}$. HMME-HA-CNT suspensions in water $(0.5 \mathrm{~mL})$ at different concentrations were illuminated with an $808 \mathrm{~nm}$ continuous-wave NIR laser with a power density of $1.4 \mathrm{~W} / \mathrm{cm}^{2}$ and a spot size of $6 \times 8 \mathrm{~mm}$ (exposure time, 10 minutes). The temperature was measured by a thermometer (HT-8878; Zhengzhou Jinyangguang Instrument, Zhengzhou, People's Republic of China).

\section{Cell-culture and viability measurements}

The B16F10 mice melanoma cell line was obtained from Chinese Academy of Sciences Cell Bank (TCM36). Cells were cultured in normal DMEM with $10 \%$ fetal bovine serum and $1 \%$ penicillin/streptomycin in $5 \% \mathrm{CO}_{2}$ and $95 \%$ air at $37^{\circ} \mathrm{C}$ in a humidified incubator.

For cell-viability measurements, B16F10 cells were plated in 96-well plates and then incubated for 24 hours. After incubation of B16F10 cells with various concentrations of free HMME, HA-CNTs, and HMME-HA-CNTs for 24 hours, the cells were or were not irradiated with $532 \mathrm{~nm}$ laser and $808 \mathrm{~nm}$ laser with power densities of $100 \mathrm{~mW} / \mathrm{cm}^{2}$ and $1.4 \mathrm{~W} / \mathrm{cm}^{2}$ for 0.5 and 1 minute. The cells were also treated with different concentrations of HA-CNTs for 24 hours to investigate the cytotoxicity of the blank delivery system alone. Standard SRB assay was carried out to determine cell viabilities.

\section{Cellular uptake}

FITC in dimethyl sulfoxide $(1 \mathrm{mg} / \mathrm{mL}, 0.1 \mathrm{~mL})$ was added to the HMME-HA-CNT nanosuspension $(5 \mathrm{~mL})$ and 
sonicated with the ultrasonic cell-disruption system to obtain FITC-HMME-HA-CNTs. Excess FITC was removed by a Sephadex G-25 column (Sigma-Aldrich). B16F10 cells were seeded at $5 \times 10^{4}$ cells per well on glass coverslips in 24-well plates. When cells reached $70 \%$ confluence, they were treated with FITC and FITC-HMME-HA-CNTs (HA-CNT concentration: $2.5-5 \mu \mathrm{g} / \mathrm{mL}$ ) for 30 minutes, and 1,2 , and 4 hours, respectively. At indicated time points, cell medium was removed and cells were washed with phosphate-buffered saline (PBS), then trypsinized and resuspended in fresh DMEM. After adjusting cell density to $1 \times 10^{6}$ cells $/ \mathrm{mL}$, the samples were analyzed by flow cytometry (Epics XL-MCL; Beckman Coulter, Brea, CA, USA).

\section{Cell-apoptosis assay}

Apoptosis was monitored by a Hoechst 33258 assay (SigmaAldrich). B16F10 cells were treated with HMME, HA-CNTs, and HMME-HA-CNTs for 24 hours (HMME dose, $10 \mu \mathrm{g} / \mathrm{mL}$; HA-CNT dose, $4.35 \mu \mathrm{g} / \mathrm{mL}$ ), and the laser irradiation was performed under the aforementioned irradiating conditions, then the cells were fixed with methanol at $-20^{\circ} \mathrm{C}$ for 10 minutes and stained with Hoechst $33258(10 \mu \mathrm{g} / \mathrm{mL}$ in distilled water for 5 minutes) to visualize DNA chromatin. After being washed and air-dried, these samples were observed under UV excitation and the number of apoptotic cells counted using fluorescence microscopy (Zeiss).

\section{Xenograft tumor mouse model}

All animal experiments were performed under a protocol approved by the Henan Laboratory Animal Center. Mouse melanoma tumor models were generated by subcutaneous injection of $1 \times 10^{6} \mathrm{~B} 16 \mathrm{~F} 10$ cells in $0.1 \mathrm{~mL}$ saline into the right shoulder of female C57 mice (18-22 g, Henan Laboratory Animal Center). The mice were used when the tumor volume reached $60-100 \mathrm{~mm}^{3}$ ( $\sim$ days after tumor inoculation).

\section{In vivo antitumor effect}

The tumor-bearing mice were divided into five groups (five mice per group), minimizing differences in weights and tumor sizes in each group. The mice were administered with: (1) saline (0.1 mL); (2) HA-CNTs, $808 \mathrm{~nm}$ laser (2.17 mg/kg, $0.1 \mathrm{~mL})$; (3) HMME, $532 \mathrm{~nm}$ laser (5 mg/kg, $0.1 \mathrm{~mL}$ ); (4) HMME-HACNTs, $532 \mathrm{~nm}$ laser (HMME dose, $5 \mathrm{mg} / \mathrm{kg}$; HA-CNT dose, $2.17 \mathrm{mg} / \mathrm{kg}, 0.1 \mathrm{~mL}$ ); and (5) HMME-HA-CNTs, 532/808 nm laser (HMME dose, $5 \mathrm{mg} / \mathrm{kg}$; HA-CNT dose, $2.17 \mathrm{mg} / \mathrm{kg}$, $0.1 \mathrm{~mL}$ ) intravenously through tail veins every 2 days, and then the tumor regions of the laser-irradiation group were irradiated with $532 \mathrm{~nm}$ laser $\left(100 \mathrm{~mW} / \mathrm{cm}^{2}, 5\right.$ minutes $)$ and $808 \mathrm{~nm}$ laser $\left(1.4 \mathrm{~W} / \mathrm{cm}^{2}, 1\right.$ minute $)$ at 3 hours postinjection. The mice were observed daily for clinical symptoms, and the tumor sizes were measured by a caliper every other day and calculated as volume $=($ tumor length $) \times(\text { tumor width })^{2} / 2$. After treatment for 8 days, the mice were killed to collect heart, liver, spleen, lung, kidney, and tumor, and the collected tissues were soaked in $10 \%$ formalin solution, embedded with paraffin for hematoxylin and eosin (H\&E) staining. Morphological changes were observed under microscope (Zeiss LSM 510).

\section{Statistical analysis}

Quantitative data are expressed as means \pm standard deviation and analyzed by use of Student's $t$-test. $P<0.05$ was considered statistically significant.

\section{Results and discussion Synthesis and characterization of HA-CNTs}

To improve the solubility and biocompatibility of CNTs, HA-derivatization was performed via a condensation of carboxyl of HA and the $\mathrm{NH}_{2}$ group of $\mathrm{NH}_{2}-\mathrm{CNT}$, and the strategy to prepare $\mathrm{NH}_{2}-\mathrm{CNT}$ s involved carboxylation of $\mathrm{CNTs}$, resulting in $\mathrm{COOH}-\mathrm{CNTs},{ }^{27}$ and then a conjugation of ethylenediamine by the reaction between the $\mathrm{NH}_{2}$ groups of ethylenediamine and $\mathrm{COOH}$ groups of $\mathrm{COOH}-\mathrm{CNTs},{ }^{25}$ as depicted in Figure 1. FT-IR results showed two bands, at $2920 \mathrm{~cm}^{-1}$ and $1540 \mathrm{~cm}^{-1}$ corresponding to CNT core (Figure 2A). ${ }^{28} \mathrm{COOH}-\mathrm{CNT}$ s were confirmed by the strong $\mathrm{C}=\mathrm{O}\left(1710 \mathrm{~cm}^{-1}\right)$ vibrations of $\mathrm{COOH}$ due to CNT molecules (Figure 2A). ${ }^{25,29}$ Compared with $\mathrm{COOH}-\mathrm{CNTs}, \mathrm{NH}_{2}-\mathrm{CNTs}$ showed disappearance of strong $\mathrm{C}=\mathrm{O}\left(1710 \mathrm{~cm}^{-1}\right)$, and new peaks of $\mathrm{N}-\mathrm{H}\left(1630 \mathrm{~cm}^{-1}, 668 \mathrm{~cm}^{-1}\right)$ vibrations and $\mathrm{C}-\mathrm{N}$ $\left(1140 \mathrm{~cm}^{-1}\right)$ vibrations indicated the attachment of $\mathrm{NH}_{2}$ to CNTs (Figure 2A). ${ }^{25} \mathrm{HA}$ grafting to CNTs was confirmed by the new peaks of $\mathrm{C}-\mathrm{O}-\mathrm{C}\left(1046 \mathrm{~cm}^{-1}, 1078 \mathrm{~cm}^{-1}, 946 \mathrm{~cm}^{-1}\right.$, and $1150 \mathrm{~cm}^{-1}$ ) of HA (Figure 2A). ${ }^{30,31}$

The relative amount of HA grafted onto the surface of CNTs was tested by TGA. CNTs were thermally stable until $700^{\circ} \mathrm{C}$ (Figure 2B), while pure HA degraded to a constant $\left(15.1 \%\right.$ ) at about $600^{\circ} \mathrm{C}$ (Figure $2 \mathrm{~B}$ ). At $600^{\circ} \mathrm{C}, \mathrm{NH}_{2}-\mathrm{CNTs}$ and HA-CNTs showed about $9.4 \%$ and $65.3 \%$ weight losses, respectively, thus the relative amount of HA grafted onto CNTs was $40.8 \%$.

HA-CNTs were stable in water, cell-culture medium, and plasma of mice over multiple weeks without significant aggregation, but CNTs, COOH-CNTs, and $\mathrm{NH}_{2}-\mathrm{CNTs}$ 


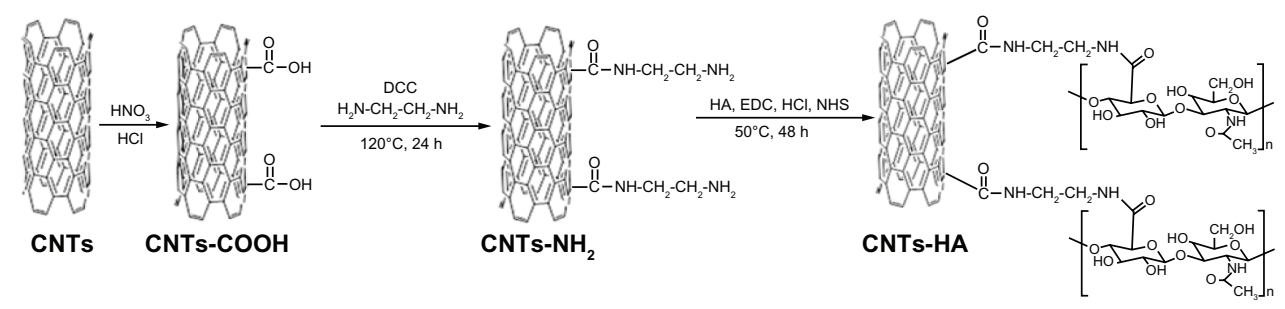

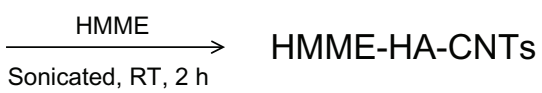

Figure I Scheme of preparation of hematoporphyrin monomethyl ether hyaluronic acid-derivatized carbon nanotubes (HMME-HA-CNTs). Abbreviations: CNT, carbon nanotubes; HMME, hematoporphyrin monomethyl ether.

were not soluble in water (Figure 2C), showing HA was successfully linked to CNTs.

\section{Preparation and characterization of HMME-HA-CNTs}

Our and others' studies have shown that aromatic molecules including many chemotherapy drugs may be loaded onto the surface of $\mathrm{sp}^{2}$ carbon nanomaterials such as CNTs and graphene by $\pi-\pi$ stacking. ${ }^{13,25,32}$ In this work, HMME, a new photodynamic anticancer drug, was loaded onto HA-CNTs via the same method. HMME loading onto HA-CNTs was confirmed by a strong absorption peak at around $395 \mathrm{~nm}$ over the HA-CNT background (Figure 3A). As expected, a significant HMME absorption in the HMME-HA-CNTs sample was observed, likely due to the close binding between HMME molecules and the CNT surface. HMME-HA-CNTs were stable in water, PBS, cell-culture medium, and plasma of mice over multiple weeks without significant aggregation (Figure 3C). To determine the adsorption equilibrium level of HMME loaded onto HA-CNTs, different feed ratios of HMME-HA-CNTs were performed, indicating that HMME loading efficiency increased from $19.8 \%$ to $240.3 \%$ (weight ratio of HMME:HA-CNTs) with the increased amount of HMME (Figure 3B). HMME loading of $~ 230 \%$ (HMME:HA-CNTs weight ratio of 3:1) was chosen for the following experiments. The amount of HMME loaded onto HA-CNTs was calculated to be $230.4 \mathrm{wt} \%$. Such a value of loading was far beyond the other CNT derivatives, ${ }^{13,33,34}$
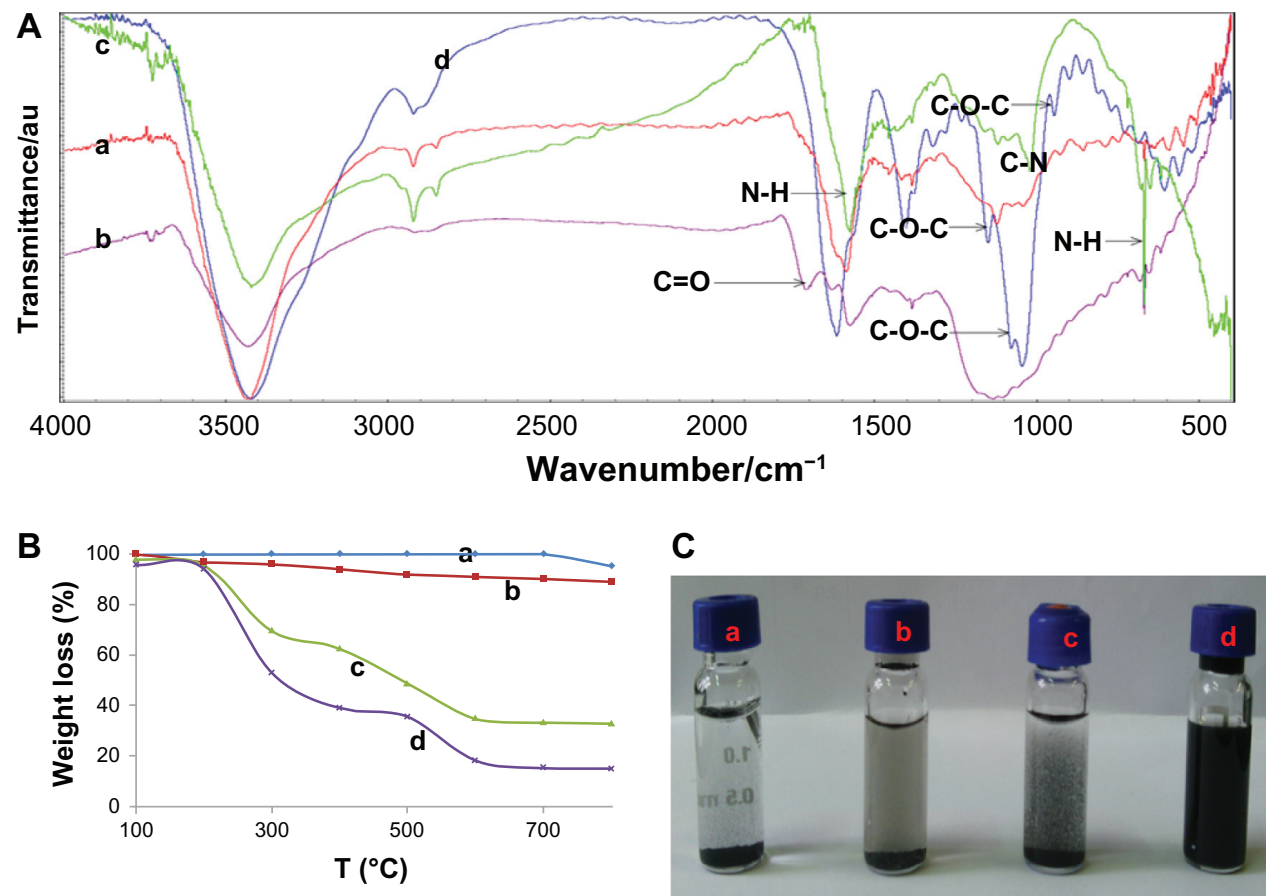

Figure 2 (A-C) Characterization of hyaluronic acid-derivatized carbon nanotubes (HA-CNTs). (A) Fourier transform infrared spectra of pristine CNTs (a), COOH-CNTs (b), $\mathrm{NH}_{2}-\mathrm{CNTs}$ (c), and HA-CNTs (d); (B) thermal gravimetric analysis curves of pristine CNTs (a), $\mathrm{NH}_{2}-\mathrm{CNTs}$ (b), HA-CNTs (c), and HA (d); (C) CNTs (a), COOH-CNTs (b), $\mathrm{NH}_{2}-\mathrm{CNTs}$ (c), and HA-CNTs (d) in water. 
A

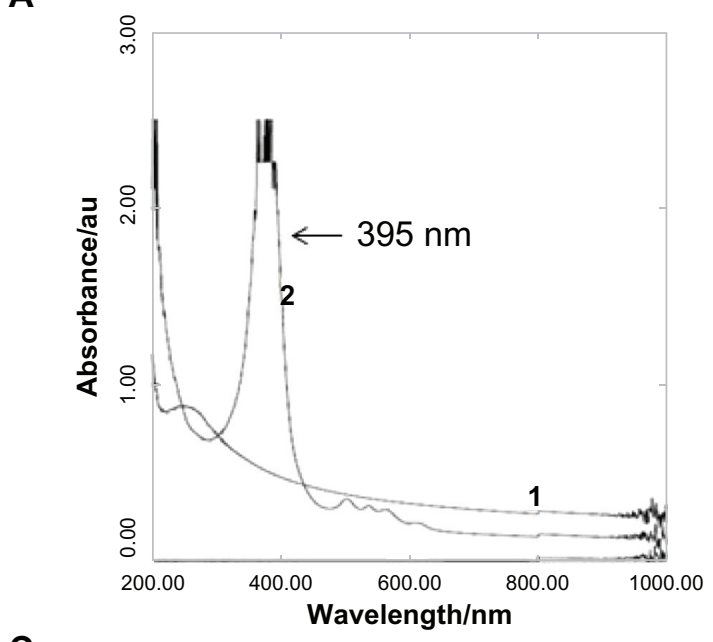

C

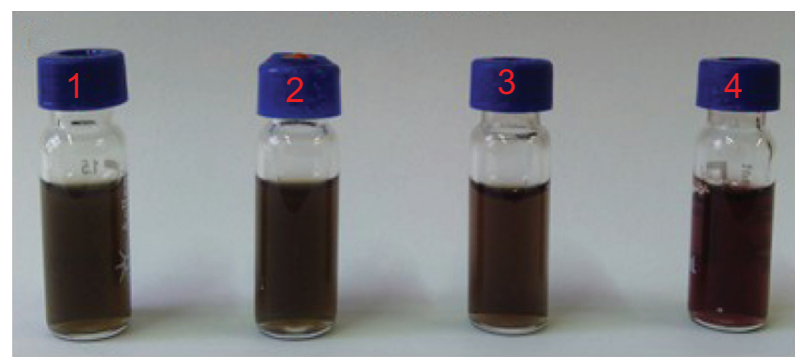

B

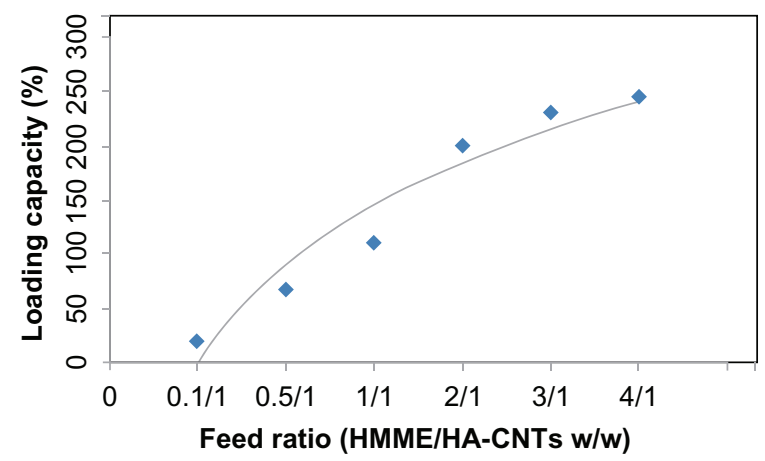

D

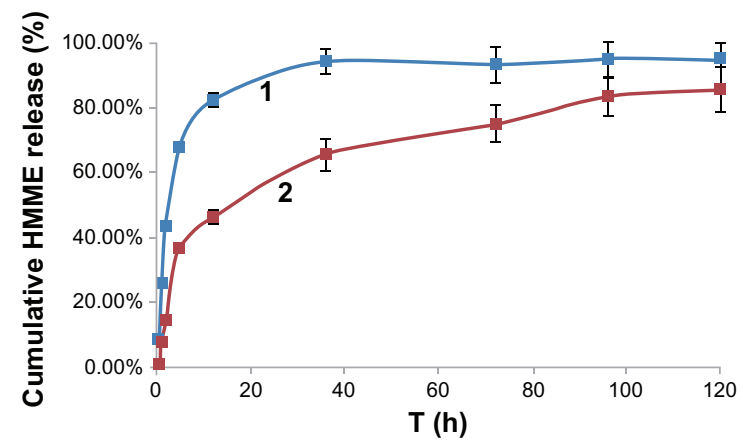

Figure 3 (A-D) Characterization of hematoporphyrin monomethyl ether hyaluronic acid-derivatized carbon nanotubes (HMME-HA-CNTs). (A) Ultraviolet spectra of HA-CNTs (I) and HMME-HA-CNTs (2) in water; (B) HMME loading at different feeding amounts of HMME; (C) photos of HMME-HA-CNTs in water (I), phosphatebuffered saline (2), plasma of mice (3) and cell culture (4) for 2 weeks; (D) Release profiles of HMME from HMME solution (I) and HMME-HA-CNTs (2).

which were always below $100 \mathrm{wt} \%$, indicating HA-CNTs were a promising material for drug delivery.

To investigate the release kinetics of active drug from the nanoparticle drug system, we incubated the nanostructures in SDS solution (0.5\%). As seen in Figure 3D, HMME release from HA-CNTs was sustained over 120 hours. In contrast, the release of the HMME group was very fast in SDS, suggesting that interaction between HA-CNTs and HMME plays a critical role in the release of drug due to a $\pi-\pi$ stacking between the surface of HA-CNTs and HMME molecules. ${ }^{35,36}$

The TEM and SEM images of HMME-HA-CNTs are shown in Figure 4A, and the zeta potential of HMME-CNTs was $-27.59 \mathrm{mV}$ (Figure 4D). The size and zeta potential of HMME-HA-CNTs were consistent with HA-CNTs, showing that HMME conjugation did not change the size or zeta potential of the drug-delivery system. From a pharmacokinetic standpoint, nanoparticles less than $5 \mathrm{~nm}$ have been reported to be cleared by the kidney, ${ }^{37}$ while larger nanoparticles have been reported to home preferentially into tumors through leaky tumor neovasculature as a result of the enhanced-permeability-and-retention (EPR) effect. ${ }^{37}$ The size of HMME-HA-CNTs opened up the possibility of targeting tumors without being cleared rapidly by the kidney.

\section{Increased temperature under NIR irradiation}

During NIR irradiation, the temperature of HMME-HA-CNT samples obviously increased from approximately $32^{\circ}$ to $68^{\circ} \mathrm{C}$ (Figure 5) as a result of the heat conversion of the NIRabsorbing HA-CNTs, while the sample with PBS displayed no obvious temperature change, revealing that HMME-HACNTs were an effective photothermal agent in cancer therapy. In addition, another control using 532 and 808 nm laser combined with a high concentration of HMME-HA-CNTs was studied, and the result showed no significant difference between HMME-HA-CNTs $808 \mathrm{~nm}$ laser and HMME-HACNTs 532/808 nm laser, indicating HMME-HA-CNTs could not generate heat under a $532 \mathrm{~nm}$ laser.

\section{Enhanced cell uptake of $\mathrm{HMME}$ by BI6FIO cells}

To explore the difference in uptake of drug by B16F10 cells, we labeled the HMME-HA-CNT nanoparticles with FITC, 

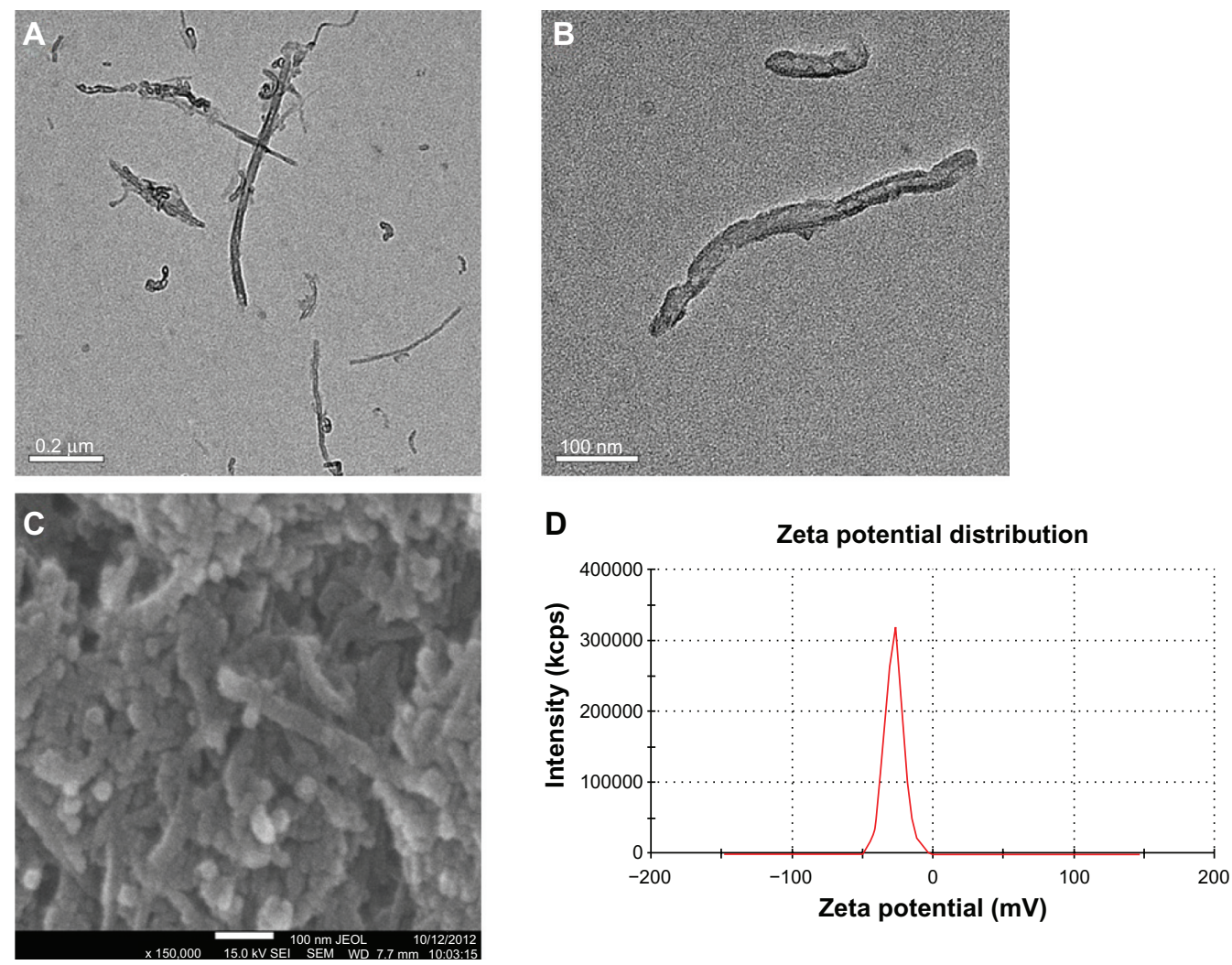

Figure 4 (A-D) Characterization of hematoporphyrin monomethyl ether hyaluronic acid-derivatized carbon nanotubes (HMME-HA-CNTs). (A and B) Transmission electron microscopy images of HMME-HA-CNTs; (C) scanning electron microscopy image of HMME-HA-CNTs; (D) zeta potential of HMME-HA-CNTs.

and tracked their internalization into the cells through colocalization of the FITC signal (green fluorescence), and then detected the changes in the FITC signal using flow cytometry (Epics XL-MCL). As shown in Figure 6, when the cells were treated with FITC alone, there was no change in the FITC signal in the cells, while the signals in FITC-HMME-HA-CNT-treated cells were obviously increased. This finding suggested that HA-CNTs could act

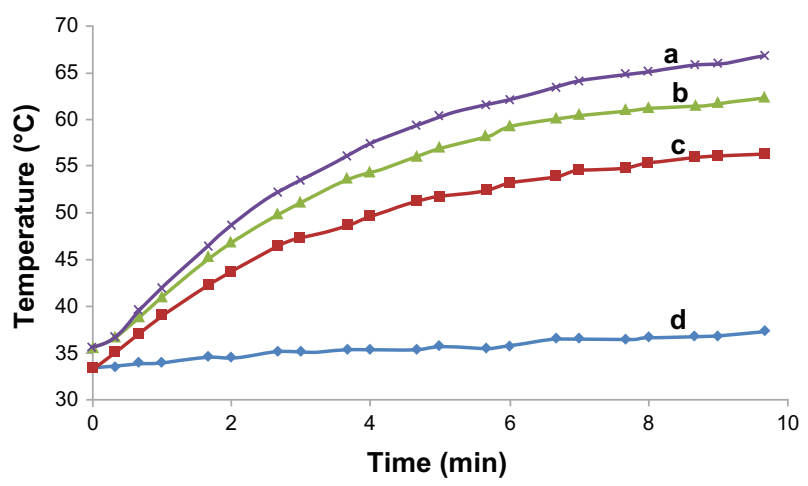

Figure 5 Temperature evolution of high (a), middle (b), and low (c) concentrations of hematoporphyrin monomethyl ether hyaluronic acid-derivatized carbon nanotubes (HMME-HA-CNTs) (HA-CNTs approximately 8.7, 6.5, and $4.3 \mu \mathrm{g} / \mathrm{mL}$; HMME approximately 20,15 , and $10 \mu \mathrm{g} / \mathrm{mL}$ ) and phosphate-buffered saline (d), during continuous radiation by $808 \mathrm{~nm}$ laser at $1.4 \mathrm{~W} / \mathrm{cm}^{2}$. as a transmembrane delivery carrier to promote the cellular uptake and increase drug intracellular accumulation. CD44 has received attention as the target receptor for cancer therapy, since it plays pivotal roles in cancer stem-like cells and resistance to chemotherapy or radiotherapy, ${ }^{38,39}$ and the HA receptor CD44 was overexpressed on B16F10 tumor cells, ${ }^{40,41}$ which made the designed drug-delivery system target B16F10 cells. The conjunction of HA to CNTs gave the HMME-CNT drug-delivery system a better susceptibility than HMME to B16F10 cells.

\section{Inhibition efficiency on BI6FIO cells}

The cytotoxicity study of HA-CNTs on B16F10 cells was carried out at different concentrations of HA-CNTs. As shown in Figure 7A, cell viability remained above $90 \%$ even at concentrations up to $100 \mu \mathrm{g} / \mathrm{mL}$. This result indicated that HA-CNTs were noncytotoxic to B16F10 cells after 24 hours' incubation. To evaluate the in vitro cytotoxicity of the free HMME, HA-CNTs, and HMME-HA-CNTs under irradiation by $532 \mathrm{~nm}$ visible light and NIR light, the viability of cells was determined by SRB assay. B16F10 cells were incubated with different concentrations of free HMME, HA-CNTs, and HMME-HA-CNTs for 24 hours. The cells 

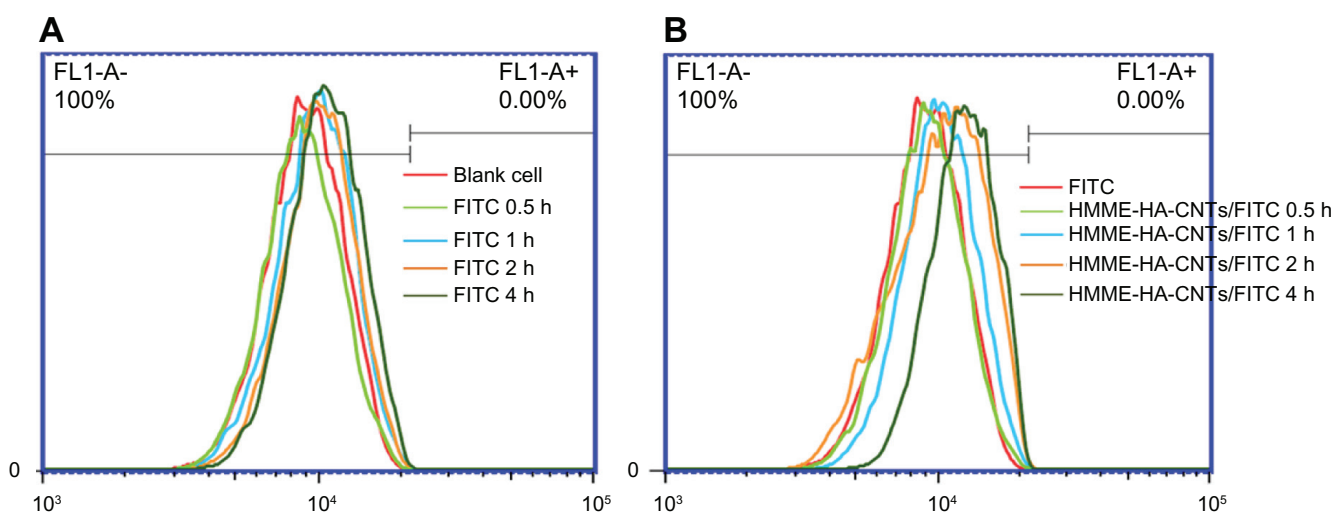

Figure 6 BI6FIO cell uptake of (A) fluorescein isothiocyanate (FITC) alone and (B) hematoporphyrin monomethyl ether hyaluronic acid-derivatized carbon nanotubes (HMME-HA-CNTs)/FITC.

treated with $808 \mathrm{~nm}$, and $532 \mathrm{~nm}$ laser as a control group was also determined, and we found the cells treated with the combined laser showed very little inhibition, indicating that the irradiating conditions that we used had no effect on cells. The HMME-HA-CNT group had an equivalent HACNT dosage to the HA-CNT group and an equal content of HMME to the HMME group. Both the HMME group and the HMME-HA-CNT group were irradiated by $532 \mathrm{~nm}$ laser (100 $\mathrm{mW} / \mathrm{cm}^{2}, 0.5$ minute), and both the HA-CNT group and
HMME-HA-CNT group were irradiated by $808 \mathrm{~nm}$ NIR laser (1.4 W/cm², 1 minute).

As seen in Figure 7B, dose-dependent cytotoxicity of all groups was shown. According to the result, we can clearly see that HMME exhibited a relatively small cytotoxicity to B16F10 cells, while the HMME with $532 \mathrm{~nm}$ laser group greatly enhanced cytotoxicity, indicating HMME is a promising photodynamic agent for cancer therapy. ${ }^{24,42}$ An advantage of PDT is that it can be positioned in treatments, ${ }^{43,44}$ so in the
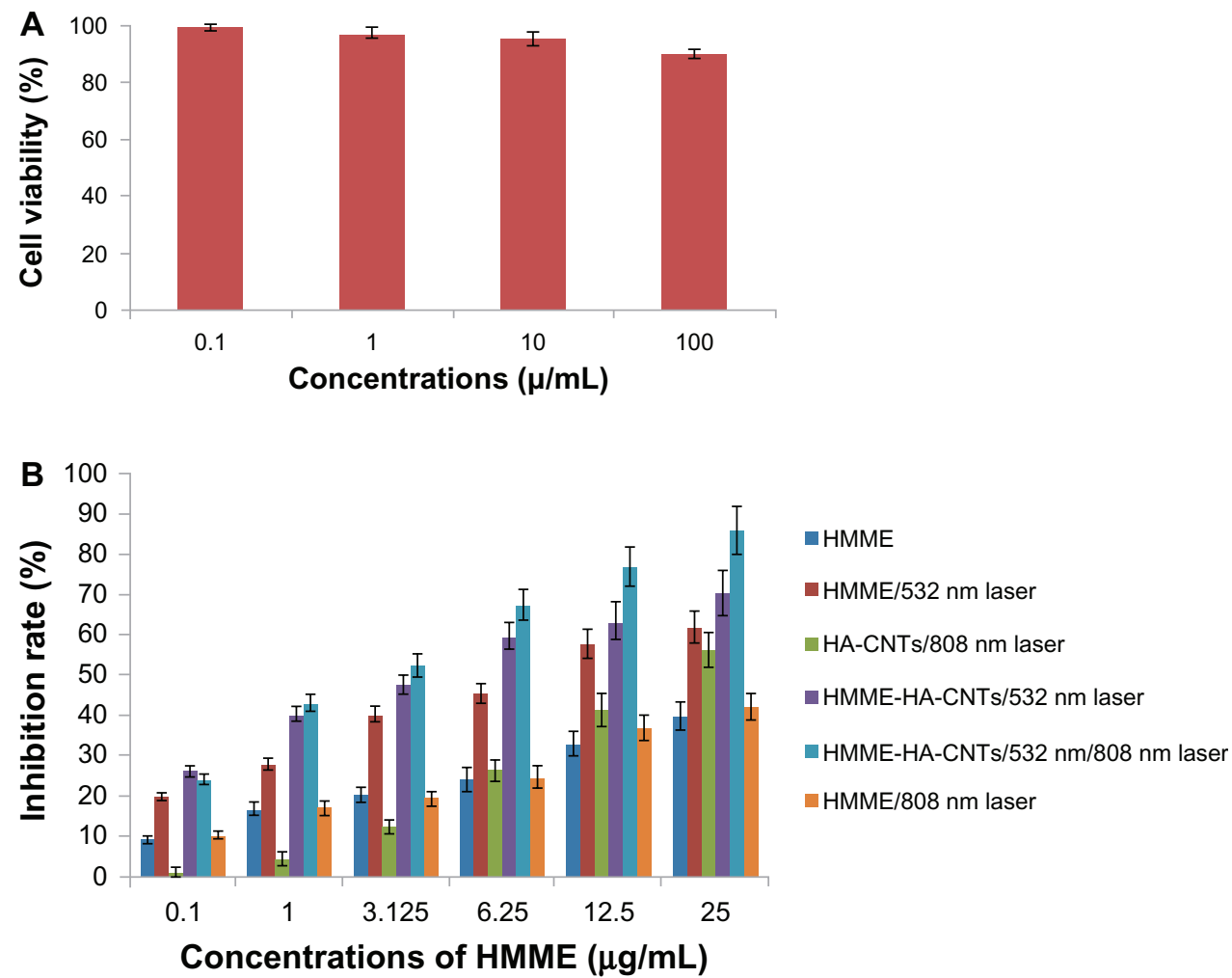

Figure 7 (A) Cell viability of BI6FI0 cells with different concentrations of hyaluronic acid-derivatized carbon nanotubes (HA-CNTs). (B) Relative cell viability of BI6FI0 cells for 24 hours after treatment with free hematoporphyrin monomethyl ether (HMME), HA-CNTs, and HMME-HA-CNTs under $100 \mathrm{~mW} / \mathrm{cm}^{2}$ of $532 \mathrm{~nm}$ laser and I.4 W/cm ${ }^{2}$ of near-infrared $808 \mathrm{~nm}$ irradiation at different concentrations.

Note: Data are presented as means \pm standard deviation $(n=6)$. 
in vivo antitumor study, we just irradiated the tumor site, and that reduced the side effects on normal tissues and organs. The result also showed HMME-HA-CNTs with $532 \mathrm{~nm}$ laser had a stronger inhibition than HMME with $532 \mathrm{~nm}$ laser, suggesting HA-CNTs could carry more HMME into cells. At an HMME concentration of $0.1 \mu \mathrm{g} / \mathrm{mL}$, the inhibition rate of HMME-HA-CNTs with $532 \mathrm{~nm}$ laser was 26.1\%, indicating higher cytotoxicity than HA-CNTs with $808 \mathrm{~nm}$ laser (1.2\%) and HMME-HA-CNTs with 532/808 nm laser (24.1\%). The lower cell-killing ability could be attributed to insufficient HA-CNTs to produce enough heat to kill cells for the HACNTs with $808 \mathrm{~nm}$ laser group and the HMME-HA-CNTs with 532/808 nm laser group. At an HMME concentration of $12.5 \mu \mathrm{g} / \mathrm{mL}$, the inhibition rate of the HMME-HA-CNTs with 532/808 nm laser significantly increased to $76.8 \%$, while HMME-HA-CNTs with $532 \mathrm{~nm}$ laser and HA-CNTs with $808 \mathrm{~nm}$ laser were $63.4 \%$ and $41.4 \%$ respectively, indicating an enhanced cell-killing effect. Furukawa et $\mathrm{a}^{45}$ reported that a sudden change of temperature from $37^{\circ} \mathrm{C}$ to $45^{\circ} \mathrm{C}$ induced a transient increase in permeability of the cell membrane, and this could lead to more drug molecules entering cells. When the HMME-HA-CNTs were exposed to the NIR laser, the CNTs were stimulated and heated to lead the temperature rise, and this could induce an increase in permeability of the B16F10 cell membranes, and lead to more HMME entering the cells, thus a synergistic therapeutic effect of PDT and photothermal therapy was observed.

\section{BI6FIO cell apoptosis}

To test whether HMME-HA-CNTs with 532/808 nm laser induce apoptosis, B16F10 cells with the treatment were stained with Hoechst 33258, and morphological changes were found in B16F10 cells incubated with HMME-HA-CNTs with 532/808 nm laser (HMME dose, $10 \mu \mathrm{g} / \mathrm{mL}$; HA-CNT dose, $4.35 \mu \mathrm{g} / \mathrm{mL} ; 532 \mathrm{~nm}$ laser, $100 \mathrm{~mW} / \mathrm{cm}^{2}$ for 0.5 minute; $808 \mathrm{~nm}$ laser, $1.4 \mathrm{~W} / \mathrm{cm}^{2}$ for 1 minute) for 24 hours. And a significant difference $(P<0.05)$ for B16F10 cell apoptosis was found between HMME-HA-CNTs with 532/808 nm laser (73.4\%) and the HMME with $532 \mathrm{~nm}$ laser group (56.8\%) (Figure 8).

\section{Inhibition of tumor growth in vivo}

To investigate in vivo therapeutic efficacy of HMME-HACNTs with 532/808 nm laser, we conducted comparative efficacy studies. The B16F10 tumor-bearing mice were divided into five groups and were treated with the protocols, as summarized in the Materials and methods section. The changes in relative tumor volume as a function of time are plotted in Figure 9A. Compared with the saline group, in
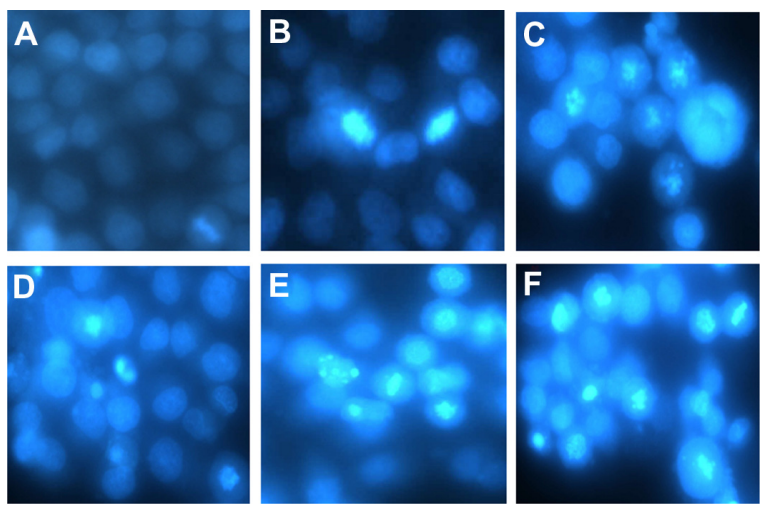

Figure 8 (A-F) Fluorescence photomicrographs of BI6FI0 cells stained with Hoechst 33258. (A) Untreated cells; (B) hematoporphyrin monomethyl ether (HMME) alone (10 $\mu \mathrm{g} / \mathrm{mL})$; (C) HMME, $532 \mathrm{~nm}$ laser ( $10 \mu \mathrm{g} / \mathrm{mL})$; (D) hyaluronic acidderivatized carbon nanotubes (HA-CNTs), $808 \mathrm{~nm}$ laser $(4.35 \mu \mathrm{g} / \mathrm{mL})$; (E) HMMEHA-CNTs, $532 \mathrm{~nm}$ laser (HMME dose, $10 \mu \mathrm{g} / \mathrm{mL}$; HA-CNT dose, $4.35 \mu \mathrm{g} / \mathrm{mL}$ ); and (F) HMME-HA-CNTs, 532/808 nm laser (HMME dose, $10 \mu \mathrm{g} / \mathrm{mL}$; HA-CNTs dose, $4.35 \mu \mathrm{g} / \mathrm{mL})$.

mice treated with HA-CNTs and $1.4 \mathrm{~W} / \mathrm{cm}^{2}$ NIR laser for 1 minute, the tumor volume was reduced, leaving black scars on the original tumor sites. Because of the EPR effect ${ }^{46,47}$ and the active tumor-targeting property of HA-CNTs, ${ }^{48,49}$ the HMME-HA-CNTs with $532 \mathrm{~nm}$ laser group could carry more HMME to the tumor site than the HMME with $532 \mathrm{~nm}$ laser group, so mice treated only with HMME with $532 \mathrm{~nm}$ laser for 5 minutes experienced a more rapid growth of tumor volume than those receiving HMME-HA-CNTs with $532 \mathrm{~nm}$ laser for 5 minutes. The growth of tumor tissue was successfully suppressed by HMME-HA-CNTs with $532 / 808 \mathrm{~nm}$ laser. The final mean relative tumor volume of mice treated with HMME-HA-CNTs with 532/808 nm laser was 1.69 , which is only $15 \%$ of the saline-treated control group (11.27). This high therapeutic efficacy originates from the high HMME accumulation and heat from CNTs by NIR irradiation in tumor tissue. Tumors of mice in HMME-HACNTs with 532/808 nm laser showed slow growth after treatment. Overall, these results not only demonstrated that HMME-HA-CNTs are highly useful for PDT of tumors but also revealed that HMME-HA-CNTs were powerful agents for combination PDT with photothermal therapy of cancer in vivo. Given that high toxicity usually leads to weight loss, we also measured the body weight of the mice for all groups during the treatments, and no weight loss was observed (Figure 9B), implying that the toxicity of treatments was not severe. Tumor-bearing mice treated with $532 \mathrm{~nm}$ laser or $808 \mathrm{~nm}$ laser are shown in Figure 10.

Therapeutic efficacy and toxicity were also evaluated by histological tissue images through H\&E staining. The H\&Estained tumor tissue in the saline group showed vigorous 

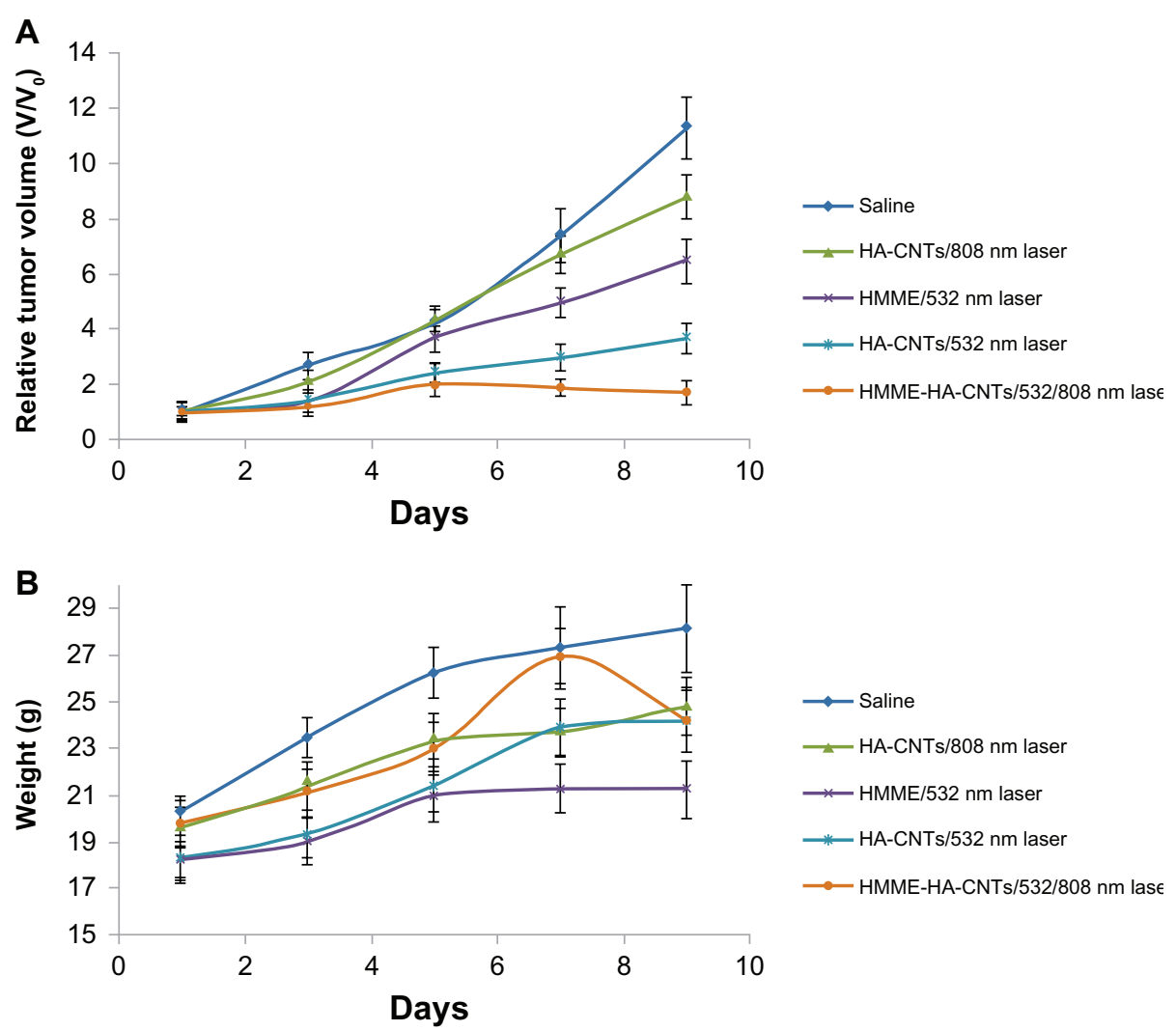

Figure 9 (A) Tumor-growth curves of different groups after treatment. The tumor volumes were normalized to their initial sizes. (B) Mean body weights of mice in different groups after treatment.

Note: Data are presented as means \pm standard deviation $(n=5)$.

Abbreviation: HMME-HA-CNTs, hematoporphyrin monomethyl ether hyaluronic acid-derivatized carbon nanotubes.

growth, a tight arrangement, a large body, and intact shape, while cell necrosis, lysis, and fragmentation occurred to a certain extent in the HA-CNTs with $808 \mathrm{~nm}$ laser, HMMEHA-CNTs with $532 \mathrm{~nm}$ laser, HMME-HA-CNTs with 532/808 nm laser, and HMME with $532 \mathrm{~nm}$ laser groups (Figure 11). A large amount of cell death in tumor tissue was observed in mice treated with HMME-HA-CNTs with
532/808 nm laser (Figure 11). However, cell death was relatively rare in the mice treated with saline, HA-CNTs with $808 \mathrm{~nm}$ laser, and HMME with $532 \mathrm{~nm}$ laser groups. No significant difference was found between control group and the treatment groups in the normal tissues.

As previously reported, ${ }^{50,51}$ combination chemotherapy with photothermal therapy based on nanoparticles demonstrated a
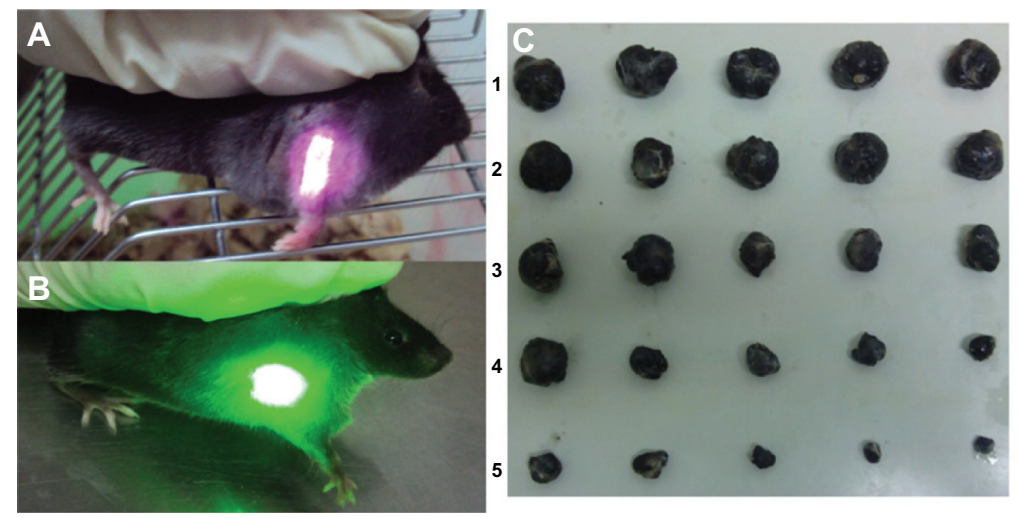

Figure 10 Tumor-bearing mice treated with (A) $808 \mathrm{~nm}$ near-infrared laser and (B) $532 \mathrm{~nm}$ laser. (C) Photo of tumors taken out of the saline group (I), the hyaluronic acid-derivatized carbon nanotubes (HA-CNTs; $808 \mathrm{~nm}$ ) laser group (2), the hematoporphyrin monomethyl ether (HMME; $532 \mathrm{~nm}$ ) laser group (3), the HMME-HA-CNT $(532 \mathrm{~nm})$ laser group (4), and the HMME-HA-CNT (532/808 nm) laser group after treatment for 8 days (5). 


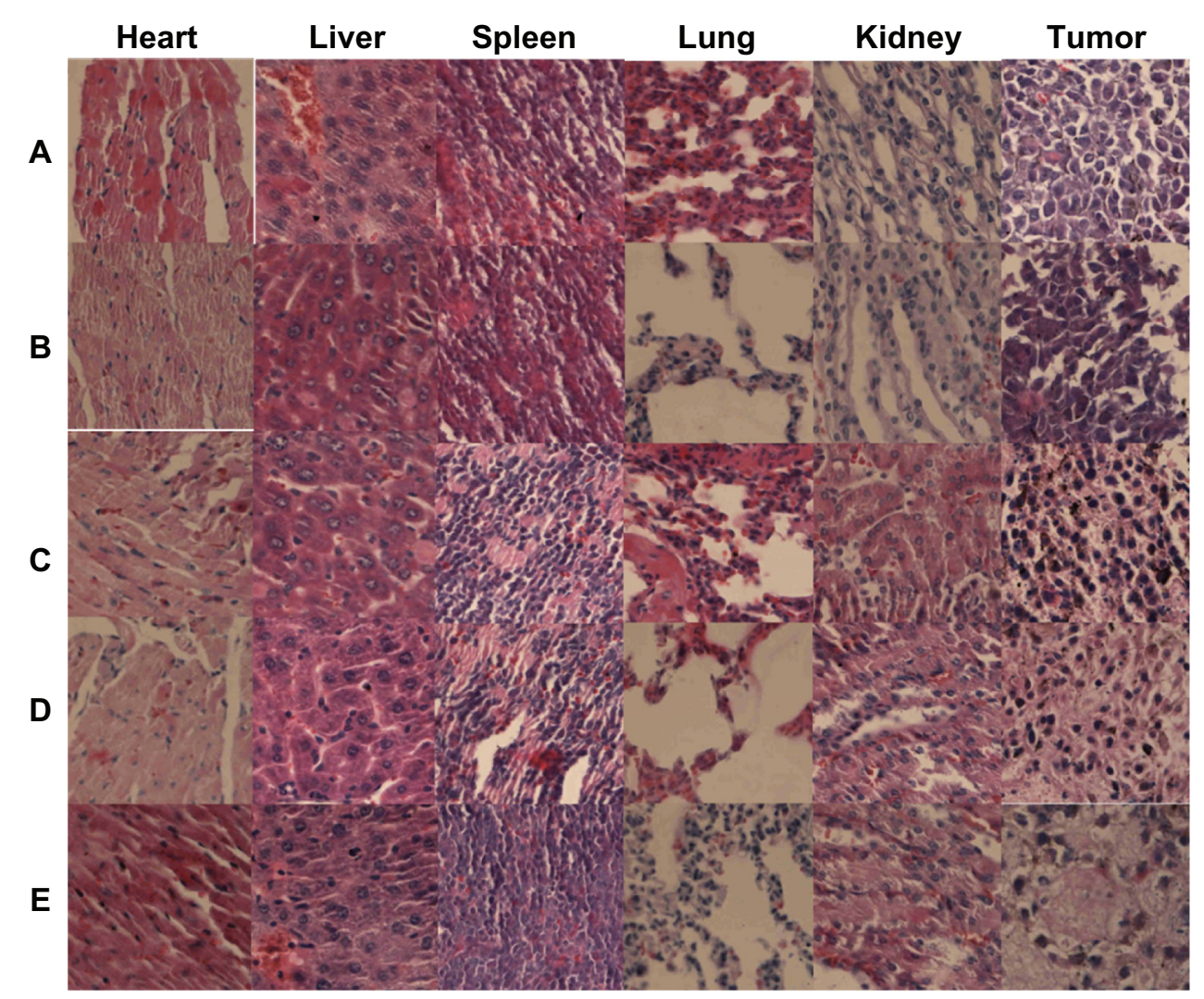

Figure I I (A-E) Histological assessments of major organs and tumor tissues with hematoxylin and eosin staining in mice (200x). (A) Saline; (B) hyaluronic acid-derivatized carbon nanotubes (HA-CNTs), $808 \mathrm{~nm}$ laser; (C) hematoporphyrin monomethyl ether, $532 \mathrm{~nm}$ laser; (D) HA-CNTs, $532 \mathrm{~nm}$ laser; and (E) HA-CNTs, $532 / 808 \mathrm{~nm}$ laser.

synergistic effect for the treatment of many solid tumors. Furthermore, because of the EPR effect, these nanoparticles can serve as drug carriers for targeting drug delivery to the tumor site, thus improving cancer therapeutic effects and reducing the nonspecific side effects of chemotherapeutics. ${ }^{52}$ On the other hand, one report ${ }^{11}$ stated that drug loaded onto CNTs could accelerate the release when the CNTs were under NIR irradiation, and in another report,$^{53}$ Chaban et al used ciprofloxacin (CIP) as a model drug loaded onto CNTs. When the CIP-CNTs were heated by NIR irradiation, the heated CNTs rapidly deposited their energy to CIP and water, increasing the diffusion coefficient of the confined CIP, and assisted the release CIP. When HMME-HA-CNTs were irradiated in the tumor site, the HMME release would be accelerated, and this perhaps is another reason for combination PDT or chemotherapy with photothermal therapy based on CNTs demonstrating a synergistic effect for the treatment of solid tumors. Furthermore, HA was a promising target to mice melanoma because its CD44 receptor overexpressed in mice melanoma cells, and this made HA-CNTs target tumors actively. The ability of higher drug-delivery efficiency to tumors by HA-CNTs was striking and directly responsible for the higher tumor-suppression efficacy of HMME-HA-CNTs with
$532 \mathrm{~nm}$ laser than the HMME with $532 \mathrm{~nm}$ laser group. The large surface area and low toxicity make HA-CNTs promising candidates for combined cancer treatment. However, much work has to be done on the in vivo behaviors of HA-CNTs, including clearance, long-term toxicology, and the mechanism of combination of the two therapies. Moreover, it is worthwhile to investigate the optimization of the drug delivery to make it more ideal for in vivo cancer treatment.

\section{Conclusion}

HA-CNTs were synthesized and the HMME-HA-CNT delivery system was successfully developed. The therapeutic effects of HMME-HA-CNTs both in vivo and in vitro combined with HMME photodynamic with HA-CNTs photothermal treatment was superior to photodynamic or photothermal treatment alone, implying that HMME-HACNTs have potential application in future tumor therapy.

\section{Acknowledgments}

The work was supported by grants from the National Natural Science Foundation of China (3097 and 3660). We wish to thank Ms Yanxia Cao (Material Department, Zhengzhou University) for her technical assistance. 


\section{Disclosure}

The authors report no conflicts of interest in this work.

\section{References}

1. Krajcik R, Jung A, Hirsch A, Neuhuber W, Zolk O. Functionalization of carbon nanotubes enables non-covalent binding and intracellular delivery of small interfering RNA for efficient knock-down of genes. Biochem Biophys Res Commun. 2008;369(2):595-602.

2. Liu Z, Chen K, Davis C, et al. Drug delivery with carbon nanotubes for in vivo cancer treatment. Cancer Res. 2008;68(16):6652-6660.

3. Chen Z, Pierre D, He H, et al. Adsorption behavior of epirubicin hydrochloride on carboxylated carbon nanotubes. Int J Pharm. 2011;405(1-2):153-161.

4. Pantarotto D, Briand JP, Prato M, Bianco A. Translocation of bioactive peptides across cell membranes by carbon nanotubes. Chem Commun (Camb). 2004;(1):16-17.

5. Kam NW, Dai H. Carbon nanotubes as intracellular protein transporters: generality and biological functionality. J Am Chem Soc. 2005;127(16): 6021-6026.

6. Kateb B, Van Handel M, Zhang L, Bronikowski MJ, Manohara H, Badie B. Internalization of MWCNTs by microglia: possible application in immunotherapy of brain tumors. Neuroimage. 2007;37 Suppl 1: S9-S17.

7. Zhang T, Tang M, Kong L, et al. Comparison of cytotoxic and inflammatory responses of pristine and functionalized multi-walled carbon nanotubes in RAW 264.7 mouse macrophages. J Hazard Mater. 2012;219-220:203-212.

8. Qiao R, Ke PC. Lipid-carbon nanotube self-assembly in aqueous solution. J Am Chem Soc. 2006;128(42):13656-13657.

9. Liu Z, Cai W, He L, et al. In vivo biodistribution and highly efficient tumour targeting of carbon nanotubes in mice. Nat Nanotechnol. 2007;2(1):47-52.

10. Heilmann K. Innovations in drug delivery systems. Curr Med Res Opin. 1983;8 Suppl 2:3-9.

11. Kam NW, O'Connell M, Wisdom JA, Dai H. Carbon nanotubes as multifunctional biological transporters and near-infrared agents for selective cancer cell destruction. Proc Natl Acad Sci U S A. 2005;102(33):11600-11605.

12. Brennan ME, Coleman JN, Drury A, Lahr B, Kobayashi T, Blau WJ. Nonlinear photoluminescence from van Hove singularities in multiwalled carbon nanotubes. Opt Lett. 2003;28(4):266-268.

13. Wang L, Zhang M, Zhang N, et al. Synergistic enhancement of cancer therapy using a combination of docetaxel and photothermal ablation induced by single-walled carbon nanotubes. Int J Nanomedicine. 2011;6: 2641-2652.

14. Fraser JR, Laurent TC. Turnover and metabolism of hyaluronan. Ciba Found Symp. 1989;143:41-53; discussion 53-49, 281-285.

15. Choi KY, Chung H, Min KH, et al. Self-assembled hyaluronic acid nanoparticles for active tumor targeting. Biomaterials. 2010;31(1): 106-114.

16. Liu Y, Sun J, Cao W, et al. Dual targeting folate-conjugated hyaluronic acid polymeric micelles for paclitaxel delivery. Int J Pharm. 2011;421(1): 160-169.

17. Toole BP, Wight TN, Tammi MI. Hyaluronan-cell interactions in cancer and vascular disease. J Biol Chem. 2002;277(7):4593-4596.

18. Brown TJ. The development of hyaluronan as a drug transporter and excipient for chemotherapeutic drugs. Curr Pharm Biotechnol. 2008;9(4):253-260.

19. Banerji S, Wright AJ, Noble M, et al. Structures of the Cd44-hyaluronan complex provide insight into a fundamental carbohydrate-protein interaction. Nat Struct Mol Biol. 2007;14(3):234-239.

20. Yoon HY, Koo H, Choi KY, et al. Tumor-targeting hyaluronic acid nanoparticles for photodynamic imaging and therapy. Biomaterials. 2012;33(15):3980-3989.
21. Nseyo UO, DeHaven J, Dougherty TJ, et al. Photodynamic therapy (PDT) in the treatment of patients with resistant superficial bladder cancer: a long-term experience. J Clin Laser Med Surg. 1998;16(1):61-68.

22. Fu QT, Gu Y, Liu FG, Zhu JG, Sun YQ, Liu AQ. Photosensitivity of HMME in vitro. Acta Laser Biol Sin. 2000;9:137-141.

23. Gu Y, Liu FG, Wang K, et al. A clinic analysis of 1216 cases of port wine stain treated by photodynamic therapy. Chin J Laser Med Surg. 2001;10:86-89.

24. Cai H, Gu Y, Zeng J, et al. Effect of HMME-PDT on hyperplastic scar in rabbit ear model. Zhonghua Zheng Xing Wai Ke Za Zhi. 2007;23(5): 425-427. Chinese.

25. Wang L, Shi J, Zhang H, et al. Synergistic anticancer effect of RNAi and photothermal therapy mediated by functionalized single-walled carbon nanotubes. Biomaterials. 2013;34(1):262-274.

26. Li P, Sun JG, Huang CR, et al. Development and validation of a sensitive quantification method for hematoporphyrin monomethyl ether in plasma using high-performance liquid chromatography with fluorescence detection. Biomed Chromatogr. 2006;20(12):1277-1282.

27. Kim D, Choi J. Water-soluble polythiophene-single walled carbon nanotube bulk heterojunction. J Nanosci Nanotechnol. 2011;11(10): 8543-8546.

28. Shang HY, Liu CG, Wei F. FT-IR study of carbon nanotube supported Co-Mo catalysts. J Nat Gas Chem. 2004;13(2):95-100.

29. Li W, Gao C. Efficiently stabilized spherical vaterite $\mathrm{CaCO}_{3}$ crystals by carbon nanotubes in biomimetic mineralization. Langmuir. 2007;23(8):4575-4582.

30. Fan DH, Wu BB, Zheng X, Gu QS. Determination of hyaluronan by spectroscopic methods. Chem Mater Sci. 2006;21(3):32-34.

31. Fagnola M, Pagani MP, Maffioletti S, Tavazzi S, Papagni A. Hyaluronic acid in hydrophilic contact lenses: spectroscopic investigation of the content and release in solution. Cont Lens Anterior Eye. 2009;32(3):108-112.

32. Liu Z, Fan AC, Rakhra K, et al. Supramolecular stacking of doxorubicin on carbon nanotubes for in vivo cancer therapy. Angew Chem Int Ed Engl. 2009;48(41):7668-7672.

33. Liu Z, Sun X, Nakayama-Ratchford N, Dai H. Supramolecular chemistry on water-soluble carbon nanotubes for drug loading and delivery. ACS Nano. 2007;1(1):50-56.

34. Murakami T, Ajima K, Miyawaki J, Yudasaka M, Iijima S, Shiba K. Drug-loaded carbon nanohorns: adsorption and release of dexamethasone in vitro. Mol Pharm. 2004;1(6):399-405.

35. Luo X, Matranga C, Tan S, Alba N, Cui XT. Carbon nanotube nanoreservior for controlled release of anti-inflammatory dexamethasone. Biomaterials. 2011;32(26):6316-6323.

36. Hampel S, Kunze D, Haase D, et al. Carbon nanotubes filled with a chemotherapeutic agent: a nanocarrier mediates inhibition of tumor cell growth. Nanomedicine (Lond). 2008;3(2):175-182.

37. Peer D, Karp JM, Hong S, Farokhzad OC, Margalit R, Langer R. Nanocarriers as an emerging platform for cancer therapy. Nat Nanotechnol. 2007;2(12):751-760.

38. Zhang L, Yao J, Zhou J, Wang T, Zhang Q. Glycyrrhetinic acid-grafthyaluronic acid conjugate as a carrier for synergistic targeted delivery of antitumor drugs. Int J Pharm. 2013;441(1-2):654-664.

39. Bourguignon LY, Spevak CC, Wong G, Xia W, Gilad E. HyaluronanCD44 interaction with protein kinase $\mathrm{C}$ (epsilon) promotes oncogenic signaling by the stem cell marker Nanog and the production of microRNA-21, leading to down-regulation of the tumor suppressor protein PDCD4, anti-apoptosis, and chemotherapy resistance in breast tumor cells. J Biol Chem. 2009;284(39):26533-26546.

40. Foureau DM, McKillop IH, Jones CP, Amin A, White RL, Salo JC. Skin tumor responsiveness to interleukin- 2 treatment and CD8 Foxp3+T cell expansion in an immunocompetent mouse model. Cancer Immunol Immunother. 2011;60(9):1347-1356.

41. Piotrowicz RS, Damaj BB, Hachicha M, Incardona F, Howell SB, Finlayson M. A6 peptide activates CD44 adhesive activity, induces FAK and MEK phosphorylation, and inhibits the migration and metastasis of CD44-expressing cells. Mol Cancer Ther. 2011;10(11):2072-2082. 
42. Jin H, Huang $X$, Chen $Y$, et al. Photoinactivation effects of hematoporphyrin monomethyl ether on Gram-positive and -negative bacteria detected by atomic force microscopy. Appl Microbiol Biotechnol. 2010;88(3):761-770.

43. Zhao T, Shen X, Li L, et al. Gold nanorods as dual photo-sensitizing and imaging agents for two-photon photodynamic therapy. Nanoscale. 2012;4(24):7712-7719.

44. Gandra N, Abbineni G, Qu X, Huai Y, Wang L, Mao C. Bacteriophage bionanowire as a carrier for both cancer-targeting peptides and photosensitizers and its use in selective cancer cell killing by photodynamic therapy. Small. 2013;9(2):215-221.

45. Furukawa M, Enomoto K, Kato H, Ishida T, Maeno T. Effects of hyperthermia on intracellular calcium concentration and responses of cancerous mammary cells in culture. Cell Biochem Funct. 1992;10(4): 225-232.

46. Oh KS, Lee H, Kim JY, et al. The multilayer nanoparticles formed by layer by layer approach for cancer-targeting therapy. J Control Release. 2013;165(1):9-15

47. Shi J, Zhang H, Wang L, et al. PEI-derivatized fullerene drug delivery using folate as a homing device targeting to tumor. Biomaterials. 2013;34(1):251-261.
48. Park K, Lee MY, Kim KS, Hahn SK. Target specific tumor treatment by VEGF siRNA complexed with reducible polyethyleneimine-hyaluronic acid conjugate. Biomaterials. 2010;31(19):5258-5265.

49. Yao J, Fan Y, Du R, et al. Amphoteric hyaluronic acid derivative for targeting gene delivery. Biomaterials. 2010;31(35):9357-9365.

50. Sherlock SP, Tabakman SM, Xie L, Dai H. Photothermally enhanced drug delivery by ultrasmall multifunctional $\mathrm{FeCo} /$ graphitic shell nanocrystals. ACS Nano. 2011;5(2):1505-1512.

51. Wu W, Shen J, Banerjee P, Zhou S. Core-shell hybrid nanogels for integration of optical temperature-sensing, targeted tumor cell imaging, and combined chemo-photothermal treatment. Biomaterials. 2010;31(29):7555-7566.

52. Langer R. Drug delivery and targeting. Nature. 1998;392(6679 Suppl): 5-10.

53. Chaban VV, Savchenko TI, Kovalenko SM, Prezhdo OV. Heat-driven release of a drug molecule from carbon nanotubes: a molecular dynamics study. J Phys Chem B. 2010;114(42):13481-13486.
International Journal of Nanomedicine

\section{Publish your work in this journal}

The International Journal of Nanomedicine is an international, peerreviewed journal focusing on the application of nanotechnology in diagnostics, therapeutics, and drug delivery systems throughout the biomedical field. This journal is indexed on PubMed Central,

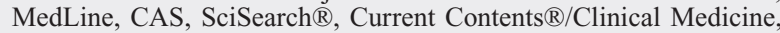

\section{Dovepress}

Journal Citation Reports/Science Edition, EMBase, Scopus and the Elsevier Bibliographic databases. The manuscript management system is completely online and includes a very quick and fair peer-review system, which is all easy to use. Visit http://www.dovepress.com/ testimonials.php to read real quotes from published authors. 